\title{
Heuristic of Possible Risks of Traffic Lights Control
}

Risk Modelling

\author{
Zurab Gasitashvili, David Satseradze, \\ Georgian Technical University, 0160, Tbilisi, st. M. Kostava 77, Georgia
}

doi: https://doi.org/10.21467/abstracts.93.28

\begin{abstract}
Today, ecological problems in megalopolises acquire great importance. One of the major factors of the negative impact on the health of population becomes the air pollution caused by permanent traffic tams due to the growing intensity and number of vehicles. Obviously, the traffic regulation proves to have decisive significance. In order to solve these problems, various intellectual transportation systems have been designed with the purpose to seek and implement the most effective ways of traffic regulation. Each of these systems has its own facilities, methods and individual approaches based on mathematical and computer technologies. All these systems have one common denominator. This is traffic problem requiring solution for each single town. Namely, necessity of modeling the intensity of traffic and road network possibility to foresee and determine their further conditions. The solution of these problems takes several stages and needs working
\end{abstract} out of various types of mathematical models, as well as individual approach for each stage.

Every system contains regulating element - traffic lights, due to which the system organizes the traffic by changing the duration of traffic lights cycles. Traffic lights are regulated by algorithms, entirely corresponding to the worked out mathematical and then computering models for a definite town. Complexity and variety of these models necessity of their individual setting stage by stage, for each town as well as growing number of automobiles, carrying the elements of undefined intensity value of the traffic, increases the risks connected with adaptability of traffic regulations and needs permanent corrections, resulting in the necessity to seek new ways of traffic regulations and their implementation in computer models.

The article studies both: the risks of traffic-lights control for adaptive regulation and possibilities of heuristic approach. Instead of traffic signals with a fixed cycle and adaptive algorithms that are used more often at intersections, it is proposed to consider the use of heuristic algorithms. For the intersection, a tuple of states (an ordered data set) is constructed in the form of a table. It contains: 1) technical characteristics of the road (width, number of lanes, throughput laid in accordance with the project); 2) structure of the traffic light cycle (number of phases, their sequence in the cycle, the duration of the main clock strokes of the phase, the duration of the intermediate clock strokes of the phase, the total duration of the traffic light cycle); 3) data on traffic (intensity, average speed, queue length in front of a traffic light, total number of vehicles passing through the intersection in one traffic light, number of vehicles turning right) collected from different detectors (induction loops, video cameras, infrared sensors) in real time.

The heuristic algorithm presents an information search algorithm for solutions in the state space of the intersection. The algorithm operates in several stages during which transitions to new states are constructed from the initial state, taking into account both the admissibility of each transition and the heuristic values. As a result of the operation of the algorithm, a state of traffic light cycles is sought in which the degree of utilization of the traffic capacity of the road (the ratio of the traffic flow intensity to the design traffic capacity of the carriageway) would be optimal.

(C) 2020 Copyright held by the author(s). Published by AIJR Publisher in "Abstracts of The Second Eurasian RISK-2020 Conference and Symposium" April 12- 19, 2020, Tbilisi, Georgia. Jointly organized by AMIR Technical Services LLC, Georgian Technical University, Institute of Geography (Kazakhstan) and Russian Institute of Petroleum Geology and Geophysics.

AijR DOI: $10.21467 /$ abstracts.93 